\title{
External cardioversion of atrial fibrillation: role of paddle position on technical efficacy and energy requirements
}

\author{
G L Botto, A Politi, W Bonini, T Broffoni, R Bonatti
}

\begin{abstract}
Aim-To define the effect of defibrillator paddle position on technical success and dc shock energy requirements of external cardioversion of atrial fibrillation.

Methods-301 patients (mean (SD) age 62 (11) years) with stable atrial fibrillation were randomly assigned to elective external cardioversion using anterolateral paddle position (ventricular apex-right infraclavicular area; group AL (151 patients)) or anteroposterior paddle position (sternal body-angle of the left scapula; group AP (150 patients)). A step up protocol was used, delivering a $3 \mathrm{~J} / \mathrm{kg}$ body weight dc shock, then a $4 \mathrm{~J} / \mathrm{kg}$ shock (maximum $360 \mathrm{~J}$ ), and finally a second 4 $\mathrm{J} / \mathrm{kg}$ shock using the alternative paddle location.
\end{abstract}

Results-The two groups were comparable for the all clinical variables evaluated. The cumulative percentage of patients successfully converted to sinus rhythm was $58 \%$ in group $A L$ and $67 \%$ in group $A P$ with low energy dc shock (NS); this rose to $76 \%$ in group $\mathrm{AL}$ and to $87 \%$ in group AP with high energy dc shock $(p=0.013)$. Thirty seven patients in group $\mathrm{AL}$ and 19 in group AP experienced dc shock with the alternative paddle position; atrial fibrillation persisted in 10/37 in group $A L$ and in $10 / 19$ in group AP. Mean dc shock energy requirements were lower for group AP patients than for group AL patients, at 383 (235) $v 451$ (287) J, p = 0.025. Arrhythmia duration was the only factor that affected the technical success of external cardioversion (successful: 281 patients, 80 (109) days; unsuccessful: 20 patients, 193 (229) days; $p<0.0001)$. The success rate was lower if atrial fibrillation persisted for $>6$ months: 29 of 37 (78\%) $v 252$ of 264 (95\%); $\mathrm{p}=\mathbf{0 . 0 0 0 1}$.

Department of Cardiology, Ospedale "Sant' Anna", Via

Napoleona 60, 22100 Como, Italy

G L Botto

A Politi

W Bonini

T Broffoni

R Bonatti

Correspondence to: Dr Botto

email: ccaec@tin.it

Accepted for publication 6 July 1999
Keywords: atrial fibrillation; cardioversion; electric countershock
Direct current defibrillation was introduced in clinical practice by Lown in the early 1960 s. $^{12}$ Cardioversion, the transthoracic application of dc shocks, remains a widely used clinical approach to terminate atrial fibrillation or flutter. ${ }^{34}$ For the procedure itself, various types of electrode may be used including traditional hand held paddle electrodes and self adhesive electrodes. ${ }^{5}$ Various chest placements have been used: the apex-anterior, apex-posterior, and anterior-posterior chest placements have been shown to be equally effective ${ }^{6-8}$ The aim of this study therefore was to determine in a prospective fashion the effect of defibrillator paddle position on technical success and dc shock energy requirements of elective external cardioversion of atrial fibrillation.

\section{Methods}

The research protocol was approved by the locally appointed ethics committee and informed consent was obtained from each patient.

The subjects of this prospective study were 301 consecutive patients who underwent elective external cardioversion for stable atrial fibrillation between August 1993 and December 1997. Standard clinical criteria were used to select patients for cardioversion. ${ }^{9}{ }^{10} \mathrm{We}$ excluded those with: haemodynamically unstable atrial fibrillation in which cardioversion needed to be performed urgently; left atrial dimension $>60 \mathrm{~mm}$ measured by $\mathrm{M}$ mode echocardiography; arrhythmia duration either $>2$ years or of unknown duration; and untreated hyperthyroidism.

The patients were sedated with propofol, and external cardioversion was performed with two self adhesive, preapplied, low impedance, disposable patch electrodes (R2 Medical System Inc, Carlsbad, California, USA). The pads used in this study consisted of foil electrodes covered by stannous chloride pre-gelled pads as the interface between the electrode and the chest wall. The backing was non-conductive and had an adhesive outer ring. ${ }^{5}$ The dc shocks, which had a damped sinusoidal waveform, were delivered by Lifepack 9 defibrillator (Physio Control Corporation, Redmond, Washington, USA) with the patient in full expiratory phase of ventilation. ${ }^{11}$ The time interval between each shock was at least three minutes. ${ }^{12}$

The patients were randomly assigned to undergo external cardioversion using either anterolateral defibrillator paddle position (ventricular apex-right infraclavicular area) (group 
Antero-lateral

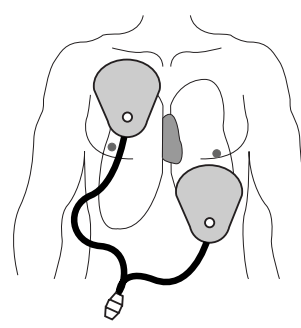

Front
Antero-posterior

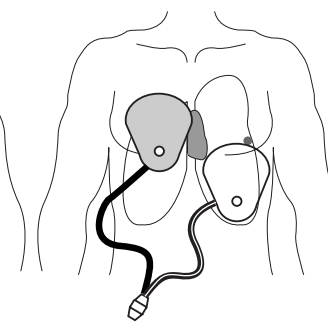

Front

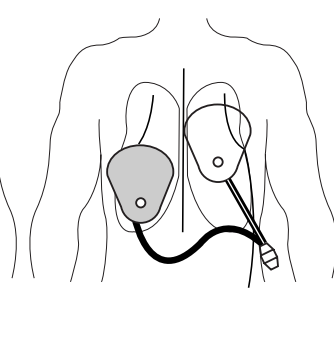

Rear

Figure 1 Electrode positions: anterolateral = ventricular apex-right infraclavicular area paddle position; (modified) anteroposterior = right sternal body at the third intercostal space-angle of the left scapula paddle position. Front, front view; rear, rear view.

AL; $\mathrm{n}=151)$ or modified anteroposterior position (right sternal body at the third intercostal space-angle of the left scapula) (group AP; $\mathrm{n}=150$ ) (fig 1 ).

A step up protocol was defined, delivering in a synchronised fashion first a $3 \mathrm{~J} / \mathrm{kg}$ body weight dc shock, then a $4 \mathrm{~J} / \mathrm{kg}$ shock (maximum $360 \mathrm{~J}$ ), and finally a second $4 \mathrm{~J} / \mathrm{kg}$ shock, relocating the electrode in the alternative position. The end point of the protocol was either the achievement of technical success or the delivery of three shocks. Technical success was defined as interruption of atrial fibrillation for $>10$ seconds.

All patients with an arrhythmia duration of $>72$ hours received anticoagulation with warfarin for at least three weeks before attempted cardioversion and continued for at least four weeks after restoration of sinus rhythm. ${ }^{13}$ Pharmacological antiarrhythmic treatment was not randomised, and some physicians began this treatment before cardioversion while others started immediately after cardioversion. All antiarrhythmic treatment before and during cardioversion was given in the steady state condition.

Data are expressed as mean (SD). Continuous variables are compared by using Student's $t$ test for independent samples. A $\chi^{2}$ test was used to determine the two tailed statistical significance of associations in $2 \times 2$ tables. A

Table 1 Patient population

\begin{tabular}{llll}
\hline & $\begin{array}{l}\text { Anterolateral group } \\
(n=151)\end{array}$ & $\begin{array}{l}\text { Anteroposterior group } \\
(n=150)\end{array}$ & $p$ Value \\
\hline Male & $94(62 \%)$ & $89(59 \%)$ & $\mathrm{NS}$ \\
Age (years) & $62(12)$ & $62(11)$ & $\mathrm{NS}$ \\
Body weight (kg) & $75(14)$ & $77(15)$ & $\mathrm{NS}$ \\
Aetiology & $39(26 \%)$ & $32(21 \%)$ & $\mathrm{NS}$ \\
$\quad$ No heart disease found & $41(27 \%)$ & $40(27 \%)$ & $\mathrm{NS}$ \\
Systemic hypertension & $42(28 \%)$ & $43(29 \%)$ & $\mathrm{NS}$ \\
$\quad$ Valvar heart disease & $14(9 \%)$ & $18(12 \%)$ & $\mathrm{NS}$ \\
$\quad$ Coronary artery disease & $15(10 \%)$ & $17(11 \%)$ & $\mathrm{NS}$ \\
$\quad$ Cardiomyopathy & $84(92)$ & $92(96)$ & $\mathrm{NS}$ \\
AF duration (days) & $75(50 \%)$ & $75(50 \%)$ & $\mathrm{NS}$ \\
Previous AF episodes & $44(6)$ & $45(6)$ & $\mathrm{NS}$ \\
Atrial diameter (mm) & $52(12)$ & $52(12)$ & $\mathrm{NS}$ \\
LVEF (\%) & & & \\
Antiarrhythmic drug treatment & $99(66 \%)$ & $97(65 \%)$ & $\mathrm{NS}$ \\
$\quad$ Total & $2(1 \%)$ & $3(2 \%)$ & $\mathrm{NS}$ \\
Quinidine & $25(17 \%)$ & $18(12 \%)$ & $\mathrm{NS}$ \\
Propafenone & $3(2 \%)$ & $1(1 \%)$ & $\mathrm{NS}$ \\
Flecainide & $62(41 \%)$ & $69(46 \%)$ & $\mathrm{NS}$ \\
Amiodarone & $7(5 \%)$ & $6(4 \%)$ & $\mathrm{NS}$ \\
Sotalol & & & \\
\hline
\end{tabular}

Values are expressed as mean (SEM) or $\mathrm{n}(\%)$.

$\mathrm{AF}$, atrial fibrillation; LVEF, left ventricular ejection fraction.

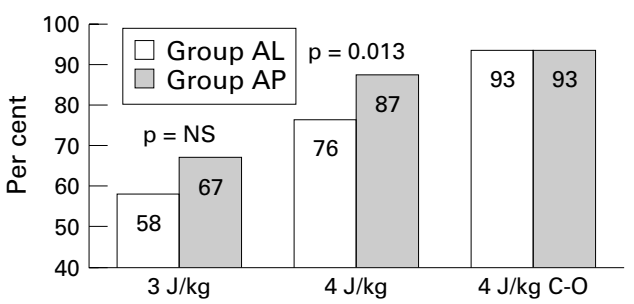

Figure 2 Success rate for each treatment group after 3 $\mathcal{F} / \mathrm{kg}$ body weight dc shock, then $4 \mathrm{~F} / \mathrm{kg}$ shock, and finally a second $4 \mathrm{f} / \mathrm{kg}$ shock after crossover of the electrode paddle position. Group AL, anterolateral paddle position; Group $A P$, anteroposterior paddle position

probability ( $\mathrm{p}$ ) value $<0.05$ was considered statistically significant.

\section{Results}

The mean age of the study population was 62 (11) years (range 26 to 80 years). The two groups were well matched, with no significant differences in age, male to female ratio, body weight, left atrial dimension, left ventricular ejection fraction, number of previous atrial fibrillation episodes, arrhythmia duration, and aetiology of heart disease (table 1). The numbers of patients who were on antiarrhythmic treatment before and during external cardioversion were also equally distributed between the two groups (the total presented in table 1 does not include patients who had been started on antiarrhythmic treatment following cardioversion). There was no significant difference in the number of patients in the two groups with no identified organic heart disease.

Overall, electrical cardioversion was effective in interrupting the arrhythmia in 281 of 301 patients $(93.4 \%)$. There were no complications related to cardioversion and in particular all the cases of bradycardia after cardioversion were transient and none required temporary pacing.

The main results are given in fig 2. Atrial fibrillation was interrupted by low energy dc shock in 87 of 151 patients (58\%) in group AL and 100 of 150 patients $(67 \%)$ in group AP (NS). With high energy dc shock, the rate of conversion rose to 114 of 151 cases (76\%) in group AL and 131 of 150 cases (87\%) in group AP $(p=0.013)$. Thus 56 patients experienced dc shock with the alternative paddle site: atrial fibrillation persisted after anteroposterior dc shock in 10 of 37 patients in group AL and after anterolateral dc shock in 10 of 19 patients in group AP.

The mean dc shock energy requirements were lower for group AP patients (383 (235) J) than for group $\mathrm{AL}$ patients (451 (287) J) $(\mathrm{p}=0.025)$.

Univariate analysis showed that the duration of atrial fibrillation episode was the only factor that affected both the technical success of external cardioversion (table 2) and the need to deliver the third dc shock. In 281 patients with successful cardioversion, arrhythmia duration was 80 (109) days, while in 20 patients with unsuccessful cardioversion it was 193 (229) days $(\mathrm{p}<0.0001)$. In 56 patients who needed the delivery of three shocks in the attempt to stop the arrhythmia, atrial fibrillation duration 
Table 2 Predictors of unsuccessful external cardioversion

\begin{tabular}{llll}
\hline & $\begin{array}{l}\text { Unsuccessful } \\
\text { cardioversion }\end{array}(n=20)$ & $\begin{array}{l}\text { Successful } \\
\text { cardioversion }(n=281)\end{array}$ & $p$ Value \\
\hline Male & $14(70 \%)$ & $169(60 \%)$ & NS \\
Age (years) & $64(10)$ & $62(12)$ & NS \\
Body weight (kg) & $75(14)$ & $76(15)$ & NS \\
Aetiology & $4(20 \%)$ & $67(24 \%)$ & NS \\
$\quad$ No heart disease & $6(30 \%)$ & $75(27 \%)$ & NS \\
Systemic hypertension & $10(50 \%)$ & $139(49 \%)$ & NS \\
Other & $193(229)$ & $80(109)$ & NS \\
AF duration (days) & $13(65 \%)$ & $137(49 \%)$ & NS \\
Previous AF episodes & $43(7)$ & $45(6)$ & NS \\
Atrial diameter (mm) & $50(13)$ & $52(12)$ & NS \\
LVEF (\%) & $13(65 \%)$ & $183(65 \%)$ & \\
Antiarrhythmic drug treatment & & &
\end{tabular}

Values expressed as mean (SEM) or $\mathrm{n}(\%)$

$\mathrm{AF}$, atrial fibrillation; LVEF, left ventricular ejection fraction.

was 133 (145) days, while in 245 patients in whom arrhythmia was interrupted with one or two shocks atrial fibrillation duration was 77 (115) days $(p=0.003)$.

When patients with arrhythmia duration longer than 180 days were compared with those with a shorter duration of arrhythmia, the conversion rate rose from $78 \%$ to $95 \%(29 / 37$ $v 252 / 264 ; \mathrm{p}=0.0001)$. The conversion rate was higher in patients with an arrhythmia duration of less than 180 days (95\%) than in those with a duration of more than 180 days (78\%) (252/264 v 29/37 patients).

\section{Discussion}

When the capacitor of the defibrillator or cardioverter discharges, the amount of current delivered depends on the impedance or resistance between the electrodes. The higher the impedance, the lower the current delivered..$^{14}$ It is the current density that traverses the muscle of the chamber to be defibrillated which determines the success of cardioversion ${ }^{16}{ }^{17}$; thus, to be successful, a critical muscle mass of the atria must be defibrillated. The current that flows is determined by the energy level selected by the operator and, because the maximum output of present defibrillators in Europe is limited to $360 \mathrm{~J}$, the principal factor that determines the success of electrical cardioversion of atrial fibrillation is transthoracic impedance to dc discharge. ${ }^{18}$ The determinants of transthoracic impedance to dc defibrillator or cardioverter discharge mainly include the interface between the electrode and skin, ${ }^{514}$ the electrode size and placement, ${ }^{141617}$ and the delivered energy. ${ }^{14}$

\section{ELECTRODE TYPE}

In the study by Kerber et al on the same self adhesive pads that we used in the present study, the transthoracic impedance was 75 (21) ohms, ${ }^{5}$ while in Ewy's study, using metal electrodes and paste (not cream or gel) between them and skin, the transthoracic impedance was significantly lower, at 56 (16) ohms. ${ }^{20}$ Despite this theoretical limitation, we obtained a very high rate of technical success using self adhesive, preapplied pads: the cumulative proportion of patients successfully converted was $93.4 \%$. This tallies well with those obtained in other series of patients (ranging from $85 \%$ to $96 \%$ ) where metal paddles were used, ${ }^{2} 82122$ and is even better than the result obtained by
Levy et $a l,{ }^{23}$ who reported a cardioversion rate of only $67 \%$ using external delivery shock. Despite this apparent advantage of metal pads, the technique of metal electrode application may also have some disadvantages: the operator requires unimpeded access to the patient's chest to apply the paddle electrode, which must be well coated with a coupling agent before use. If the gel, cream, or paste used for this purpose is inadvertently spread across the chest, the current may follow this low resistance pathway rather than traversing the thorax, shunting the current away from the heart and thus leading to failure to achieve cardioversion. ${ }^{24}$ Finally, the use of metal hand held paddle electrodes limits their use to the apex-anterior position, while with self adhesive pads any other location is obtainable.

\section{ELECTRODE LOCATION}

Optimal electrode position is important to defibrillate a critical mass of the atrium for cardioversion to occur. A variety of chest placements has been used to defibrillate the atria and although the standard placement is ventricular apex-right infraclavicular area, other placements have also been advocated. ${ }^{9}$ For example Lown reported that the anteroposterior paddle position was more effective for cardioversion of atrial fibrillation than the anterolateral position. ${ }^{1-4}$ In contrast, other investigators have reported that electrode position made only minor differences to cardioversion success. ${ }^{578}$ We used a modified anteroposterior paddle position, locating the front paddle just to the right (instead of the left) of the sternal body, and our data are in complete agreement with those previously published by Lown et al. The modified anteroposterior paddle location is superior to the anterolateral position not only in terms of technical success of cardioversion of atrial fibrillation $(87 \% v$ $76 \%$ with high energy dc shock), but also concerning the energy requirements to achieve successful cardioversion (383 (235) $v 451$ (287) J). For successful cardioversion, the current vector must transverse a critical mass of atrial muscle and the anteroposterior paddle position best fulfils this criterion; the anterolateral paddle position probably does not provide the optimal current vector and flow through the atria. However, in nine patients in whom the anteroposterior paddle position was ineffective, the arrhythmia was terminated using an anterolateral location. Therefore, depending on the individual patient, one or other of these positions may turn out to be more effective, so if the initial shocks are unsuccessful in terminating the arrhythmia the operator should consider relocating the electrode and repeating the shocks.

\section{ENERGY DELIVERED}

Various studies have examined the energy required for successful dc cardioversion. . $^{-3} 5212526$ Cardioversion using $100 \mathrm{~J}$ will only be successful in about $50 \%$ of patients with atrial fibrillation, ${ }^{521}$ while this figure rises to about $75-85 \%$ with $200 \mathrm{~J} .{ }^{1-3} 212526$ 
Though it is desirable to use the lowest energy shock likely to restore sinus rhythm, in order to avoid deleterious myocardial and haemodynamic effects, ${ }^{27}$ we decided that the initial energy delivered in the present study had to be in the middle energy range (that is, $200 \mathrm{~J}$ for a $66 \mathrm{~kg}$ patient) because our aim was to reduce the total number of shocks. The next shock was a high energy shock (maximum 360 $\mathrm{J})$ because it has been shown that almost all patients in whom external cardioversion can be achieved will respond to the maximum setting. ${ }^{1-3}$ If the first high energy shock was ineffective, we delivered a final high energy shock with a different electrode paddle location. This protocol allowed an increase in the overall success rate from $81 \%$ after the second shock to $93 \%$ after the third shock, an increase of $15 \%$. However, our finding of increased efficacy with the third shock contrasts with the results of a previous study in a smaller number of patients in which the delivery of a third shock did not increase the success rate of cardioversion..$^{22}$ In this latter study the electrode position was unchanged between the second and the third shock.

\section{CLINICAL VARIABLES}

Of all the variables studied, duration of atrial fibrillation was the best predictor of technical failure of external cardioversion. The mean arrhythmia duration was 80 (109) days in patients with successful cardioversion, and 193 (229) days in those with unsuccessful cardioversion, while the success rate was reduced by $18 \%$ $(78 \% v 95 \%)$ if atrial fibrillation had persisted for more than six months before cardioversion. This confirms the results of a previous report. ${ }^{9}$ It has been shown that paroxysms of atrial fibrillation lead to changes in atrial electrophysiological properties in animals ${ }^{28}$ and humans. ${ }^{29}$ Prolonged maintenance of atrial fibrillation by a fibrillatory pacemaker leads to long term shortening of the atrial refractory period in the absence of any significant effects on intra-atrial conduction velocity. ${ }^{28} 30$ This electrical remodelling is thought to promote the progressive persistence of this arrhythmia. It is thus possible that the modification of atrial electrophysiological parameters could make the interruption of the arrhythmia more difficult.

In contrast to a previous study, ${ }^{31}$ we failed to demonstrate any value of $M$ mode measurement of atrial size as a predictor of unsuccessful cardioversion, suggesting that patients with larger atrial sizes (up to at least $60 \mathrm{~mm}$ ) should still be considered for cardioversion.

\section{LIMITATIONS}

This study did have a limitation. Although it was prospective and included a relatively large number of patients, the antiarrhythmic drug treatment was not randomly assigned, but prescribed individually according to the patient's history. This might have accounted for the lack of any difference in cardioversion success between patients who were on antiarrhythmic drugs at the time of cardioversion and those who were not.
CONCLUSIONS

The anteroposterior (right anterior, left posterior) defibrillator paddle position is superior to the anterolateral location in terms of the technical success of elective external cardioversion of stable atrial fibrillation, and allows lower dc shock energy requirements. However, in some patients the anterolateral position could also prove to be more effective. Thus if initial shocks are unsuccessful in terminating the arrhythmia, the operator should consider relocating the electrode and repeating the shocks. The high success rate cardioversion obtained using this protocol should minimise the need for internal cardioversion for converting atrial fibrillation, ${ }^{32}$ limiting this procedure to those patients with atrial fibrillation who have failed conventional external cardioversion. ${ }^{33}$

The data from this study could be used when considering cardioversion in patients with long standing atrial fibrillation.

1 Lown B, Amarasinghem R, Neuman J. New method for terminating cardiac arrhythmias: use of synchronized capacitor discharge. $\mathcal{F A M A}$ 1962;182:548-55.

2 Lown B, Perlroth MG, Bey SK, et al. Cardioversion of atrial fibrillation. A report on the treatment of 65 episodes in 59 patients. N Engl f Med 1963;269:325-31.

3 Lown B. Electrical reversion of cardiac arrhythmias. $\mathrm{Br}$ Heart f 1967;29:469-89.

4 De Silva RA, Graboys TB, Podrid PJ, et al. Cardioversion and defibrillation. Am Heart f 1980;100:881-95.

5 Kerber RE, Martins JB, Kelly KJ, et al. Self-adhesive preapplied electrode pads for defibrillation and cardioversion. $\mathcal{F}$ Am Coll Cardiol 1984;3:815-20.

6 Kerber RE, Grayzel J, Kennedy J, et al. Electrical cardioversion: influence of paddle electrode location and size on success rates and energy requirements. $N$ Engl 7 Med 1981;305:658-62.

7 Mathew TP, Moore A, McIntyre M, et al. Randomized comparison of antero-apical and antero posterior routes for cardioversion of atrial fibrillation [abstract]. Circulation 1997;96:I-74

8 Tuinenburg AE, Van Gelder IC, Tieleman RG, et al. No differences in efficacy and atrial fibrillation of the anterolateral versus the antero-posterior paddle position during external DC-countershock for atrial arrhythmia [abstract]. Eur Heart f 1997;18:540

9 Kerber RE. Transthoracic cardioversion of atrial fibrillation and flutter: standard techniques and new advances. $A m \mathcal{F}$ Cardiol 1996;78(suppl 8A):22-6.

10 Costeas C, Kassotis J, Blitzer M, et al. Rhythm management in atrial fibrillation with a primary emphasis on pharmacological therapy. Part 2. PACE 1998;21:742-52.

11 Ewy GA, Hellman DA, McClung S, et al. Influence of ventilation phase on transthoracic impedance and defibrillatilation phase on transthoracic impedance and

12 Dahl CF, Ewy GA, Ewy MD, et al. Transthoracic Dahl CF, Ewy GA, Ewy $\mathrm{MD}$, et al. Transthoracic
impedance to direct current discharge: effects of repeated impedance to direct current discharge: effects

13 Laupacis A, Albers G, Dalen J, et al. Antithrombotic therapy in atrial fibrillation. Chest 1995;108:352-9S.

4 Connel PN, Ewy GA, Dahl CF, et al. Transthoracic impedance to defibrillator discharge: effect of electrode size and chest wall interface. $\mathcal{F}$ Electrocardiogr 1973;6:313.

15 Ewy GA. Direct current shock and transcardiac impedance [letter]. Am f Cardiol 1980;45:909.

16 Thomas ED, Ewy GA, Dahl CF, et al. Effectiveness of direct current defibrillation: role of paddle electrode size. $A m$ Heart 7 1977;93:463-7.

17 Ewy GA. Effectiveness of direct current defibrillation: role of paddle electrode size, II [letter]. Am Heart f 1977;93:674-5. 18 Ewy GA. Optimal technique for electrical cardioversion of atrial fibrillation. Circulation 1992;86:1645-7.

19 Ewy GA, Taren D. Comparison of paddle electrode pastes Ewy GA, Taren D. Comparison of paddle electrode
used for defibrillation. Heart Lung 1977;6:847-50.

20 Ewy GA. Cardiac arrest and resuscitation: defibrillators and defibrillation. Curr Prob Cardiol 1978;11:1-71.

21 Ricard P, Lèvy S, Trigano J, et al. Prospective assessment of the minimum energy needed for external electrical cardioversion of atrial fibrillation. Am f Cardiol 1997;79:815-16.

22 Sermasi S, Marconi M, Cioppi F. Cardioversione elettrica di elezione della fibrillazione atriale non isolata dell' adulto: proposta di ottimizzazione della procedura. G Ital Cardiol 1995;25:1399-406.

23 Lévy S, Lauribe P, Dolla E, et al. A randomized comparison of external and internal cardioversion of chronic atrial fibrillation. Circulation 1992;86:1415-20.

24 Caterine MR, Yoerger DM, Spencer KT, et al. Are paddle electrode position and gel technique important for transthoracic defibrillation? [abstract] Circulation 1994;90:I-287.

25 Kerber RE. Energy requirements for defibrillation. Circulation $1986 ; 74: 117-19$. 
26 Kerber RE, Martins JB, Kienzle MG, et al. Energy, current and success in defibrillation and cardioversion: clinical studies using an automated impedance-based method of energy adjustment. Circulation 1988;77:1038-46.

27 Ditchey RV, LeWinter MM. Effects of direct current electrical shocks on systolic and diastolic left ventricular function in dogs. Am Heart 7 1983;105:727-31.

28 Wijffels MCEF, Kirchhof CJHJ, Dorland R, et al. Atrial fibrillation begets atrial fibrillation. A study in awake chronically instrumented goats. Circulation 1995;92:195468.

29 Daoud EG, Niebauer M, Bogun F, et al. Atrial fibrillation induces shortening of refractoriness in humans [abstract]. Circulation 1995;92:401.
30 Goette A, Honeycutt G, Langberg JJ. Electrical remodeling in atrial fibrillation: time course and mechanisms. Circulain atrial fibrillation: tim
tion 1996;94:2968-74.

31 Henry WL, Morganroth J, Pearlman AS, et al. Relation between echocardiographically determined left atrial size and atrial fibrillation. Circulation 1976;53:273-9.

32 Murgatroyd FD, Slade AKB, Sopher M, et al. Efficacy and tolerability of transvenous low energy cardioversion of paroxysmal atrial fibrillation in humans. $\mathcal{f} \mathrm{Am}$ Coll Cardiol 1995;25:1347-53

33 Schmitt C, Alt E, Plewan A, et al. Low energy intracardiac cardioversion after failed conventional external cardioversion of atrial fibrillation. F Am Coll Cardiol 1996;28:994-9.

\section{Electronic Pages}

\section{eHEART: www.heartjnl.com}

The following electronic only articles are published in conjunction with this issue of Heart.

\section{Physical activity is a major contributor to the ultra low frequency components of heart rate variability f $M$ Serrador, H C Finlayson, $R$ L Hughon}

Objective-To investigate the link between changes in level of physical activity and the pattern of heart rate variability during long term ambulatory monitoring.

Design - Heart rate variability was measured simultaneously with a quantitative indicator of muscle activity by electromyography (EMG) in five men and five women while they did activities typical of daily life or while they rested for 2-3 hours. Spectral and cross spectral analyses were performed on both variables with standard fast Fourier transform

Results - There was a marked reduction in spectral power in the ultra low frequency band $(<0.003 \mathrm{~Hz})$ on going from active to rest conditions for both heart rate variability (men 6187 (1801) v $410(89) \mathrm{ms}^{2} / \mathrm{Hz}$; women 4056 (1161) v 2094 (801), mean (SEM); p < 0.01) and EMG $(\mathrm{p}<0.001)$. Cross spectral analysis showed a strong positive gain between the EMG and heart rate variability signal that was virtually eliminated in the resting condition $(\mathrm{p}<0.01)$. A sex-by-condition effect $(\mathrm{p}=0.06)$ was noted with a reduction in total spectral power for heart rate variability during rest in men, while it increased slightly in women.

Conclusions - There is a quantitative link between muscle activation and heart rate variability in the lowest frequency band. Voluntary restriction of physical activity in healthy young subjects caused marked reduction in spectral power in the lowest frequency band which is often used to assess patient prognosis. The findings strongly suggest that studies of ambulatory heart rate variability should always include an indication of physical activity patterns. (Heart 1999;82:e9)

www.heartjnl.com/cgi/content/full/82/6/e9

Aortic balloon dilatation for congenital aortic stenosis: report of 90 cases (1986-98)

A Borghi, $G$ Agnoletti, $O$ Valsecchi, $M$ Carminati

Objective-To review 12 years of experience of balloon aortic valvoplasty in childhood.

Design-Early and mid-term clinical and instrumental evaluation of 104 consecutive balloon aortic valvoplasties performed from 1986 to 1998.
Setting-A tertiary referral centre for congenital heart disease.

Patients - 90 patients with congenital aortic stenosis: 20 neonates (group 1), 16 infants (group 2), and 54 children (group 3).

Interventions-Balloon aortic valvotomy.

Main outcome measures-Doppler and peak to peak aortic gradient before and after valvoplasty, degree of aortic regurgitation before and after valvoplasty, early and late mortality, need for repeat intervention or surgery.

Results-Balloon aortic valvoplasty produced a gradient reduction of $>50 \%$ in 59 patients, 12 having a residual peak to peak gradient of $>50 \mathrm{~mm} \mathrm{Hg}$. Early mortality included three procedure related and six procedure unrelated deaths. There were no intraprocedural deaths. Grade III aortic regurgitation occurred in 20 patients. Five non-lethal complications occurred. At a mean follow up of 5.1 (group 1), 5.7 (group 2), and 7.6 years (group 3), survival was $75 \%$, $88 \%$, and $96 \%$, respectively. Redilatation was performed in three patients in group 1, one in group 2, and 10 in group 3. Surgery was necessary for six in group 1 , one in group 2 , and eight in group 3. Freedom from events at last follow up was $50 \%, 75 \%$, and $64 \%$, respectively. There was a residual maximum Doppler gradient of $<30 \mathrm{~mm} \mathrm{Hg}$ in 22 patients and $>60 \mathrm{~mm} \mathrm{Hg}$ in 23; 50 patients have mild to moderate aortic regurgitation.

Conclusions-Balloon aortic valvoplasty is effective and repeatable and offers good palliation for congenital aortic stenosis in childhood. (Heart 1999;82:e10)

www.heartjnl.com/cgi/content/full/82/6/e10

\section{A young man with a heavy heart \\ P Davey, Benson}

A 34 year old man presented with acute chest pain. His ECG was very abnormal but stable and he was treated with opiate analgesia. When his condition did not improve, chest radiography and cardiac ultrasound were performed. Both revealed metal dense deposits in the heart. On questioning, the patient revealed that he had self injected with mercury 15 years before. Self injection of elemental mercury is rare but well described and normally used by those who are suicidally depressed or who seek to improve sexual or athletic performance. Intravenous mercury may be deposited in the right heart and can result in ECG abnormalities, which may later be mistaken for changes due to coronary or other cardiac disease and result in inappropriate medication and hospitalisation. (Heart 1999;82:e11) www.heartjnl.com/cgi/content/full/82/6/e11 